\section{Comparison of intraocular pressure measurement by three tonometers, the TonopenXL, Tonovet and TonovetPlus in the eyes of 50 dogs with a range of normal and abnormal intraocular pressures}

\section{Natalia Giannakopoulou, David Williams}

University of Cambridge Veterinary School, Cambridge United Kingdom

\section{OBJECTIVES}

Intraocular pressure (IOP) measurement is important in ophthalmic examinations being achieved by use of applanation tonometers such as the Tonopen or rebound tonometers such as the Tonovet or more recently the Tonovet-plus. Here we seek to determine correlation between iop as measured by these tonometers.

\section{METHODS}

The Tonovet, Tonovet-plus and Tonopen were used to measure iop in eyes of 50 dogs with normal eyes (iop between 10 and $25 \mathrm{mmHg}$ ), glaucomatous (iop > $25 \mathrm{mmHg}$ ) and uveitic (iop $<10 \mathrm{mmHg}$ ) eyes. Comparison of iop values was achieved using analysis of variance (ANOVA).

\section{RESULTS}

Descriptive statistical analysis and ANOVA of measurements for all eyes showed iop values for Tonopen, Tonovet and Tonovet-plus of $20.0 \pm 11.4,22.2 \pm 12.8$ and $25.7 \pm 14.2 \mathrm{mmHg}$, an $\mathrm{f}$-ratio value of 2.52 and $p$ of 0.084 . For the 20 eyes with uveitis the iop measures were $9.85 \pm 3.39,10.45 \pm 4.54$ and $13.65 \pm 5.01 \mathrm{mmHg}$ and the $\mathrm{f}$-ratio value was 4.4 at $p<0.05$. For the 36 glaucomatous eyes iop measures were $43.89 \pm 12.11,47.72 \pm 14.38$ and $52.86+15.53 \mathrm{mmHg}$ with an f-ratio value of 3.68 at $\mathrm{p}<0.05$. For the 42 normal eyes iop measures were $16.93 \pm 2.53,18.69 \pm 3.34$ and $21.26 \pm 3.28 \mathrm{mmHg}$ with f-ratio value of 21.1 and significance at $p<0.0001$. In all cases iop measured higher using the Tonovet-plus than the Tonovet with the Tonopen providing the lowest measurements.

\section{STATEMENT (CONCLUSIONS)}

That different tonometers give different iop values does not invalidate any particular device; correlation with manometric measurement is the only method of determining measurement validity. Measurement with different tonometers did not change a diagnosis of glaucoma or uveitis, however this study does show that comparisons between tonometers is not appropriate.

\section{Al and the eye!}

\section{David Williams, Richard Dybowsky}

Department of Veterinary Medicine, University of Cambridge, Cambridge, United Kingdom

\section{OBJECTIVES}

The rise of artificial intelligence (AI) in medical diagnosis has been rapid-a PubMed search of 'artificial intelligence, clinical diagnosis and medicine' gave 7225 results. Veterinary medicine has not been as quick on the uptake. A similar PubMed search in veterinary medicine gives only 22 relevant publications. Yet the use of pattern recognition and flowchart methodology renders veterinary diagnosis quite as amenable to computer-aided modelling as is human medicine. Here we sought to develop an Al system for the diagnosis of the causes of canine red eye.

\section{METHODS}

An Al system for diagnosis that can run on a standard laptop was developed by clinical veterinary ophthalmologists in conjunction with researchers in mathematics and computational biology.

\section{RESULTS}

The Al system developed is based on a Bayesian network in which each node of the network corresponds to an attribute of interest e.g., 'breed', 'disease', "'intraocular pressure'. An arrow from one node to another indicates that the value taken by one node influences the value taken by the other; thus, 'breed' > 'disease' indicates that the probability of a dog having a particular disease is influenced by the breed. 'disease' > 'intraocular pressure' indicates that the probability a particular level of intraocular pressure is influenced by which disease is present. The network enables clinicians to evaluate which disease is likely to be manifest by specific clinical signs observed and allows students to learn the probabilistic causal relationships between eye diseases and their clinical signs.

\section{STATEMENT (CONCLUSIONS)}

This system illustrates the value of Al in clinical diagnosis and veterinary education 